\title{
Classification of some nilpotent class of Leibniz superalgebras
}

\author{
L.M. Camacho, J.R. Gómez, R.M. Navarro, B.A. Omirov
}

\begin{abstract}
The aim of this work is to present the description of Leibniz superalgebras up to isomorphism with characteristic sequence $(n \mid m-1,1)$ and nilindex $n+m$.

2000 MSC: 17A32, 17B30

Keywords: Lie superalgebras, Leibniz superalgebras, nilindex, charasteristic sequence.
\end{abstract}

\section{Introduction}

During many years the theory of Lie superalgebras has been actively studied by many mathematicians and physicists. Many works have been devoted to them but few have dealt with nilpotent Lie superalgebras. Recent works [5]-[9] have studied the problems of description of some classes of nilpotent Lie superalgebras. It is well known that Lie superalgebras are a generalization of Lie algebras [10]. In the same way, the notion of Leibniz algebras can be generalized by means of Leibniz superalgebras. The elementary properties of Leibniz superalgebras were obtained in [1]. The description of case of maximal nilindex for nilpotent Leibniz superalgebras (nilpotent Leibniz superalgebras distinguished by the feature of being single-generated) is not difficult and was done in [1]. However, the next stage is very problematic, it consists of Leibniz superalgebras with dimensions even and odd parts equal to $\mathrm{n}$ and $\mathrm{m}$, respectively, and of nilindex $n+m$. It should be noted that such Lie superalgebras were classified in [8]. Due to the great difficulty in solving the problem, some restrictions on the characteristic sequence are added. The experience of using the characteristic sequence in Lie and Leibniz algebras (even in Lie superalgebras) leads us to choose a restriction on this invariant. Since graded non-commutative identity in non Lie Leibniz superalgebras does not hold, we usually have to solve many technical tasks when describing Leibniz superalgebras, 3].

In a similar way to Leibniz algebras and Lie superalgebras cases, it is possible to define the notions of null-filiform and filiform Leibniz superalgebras [2], [5] as superalgebras with characteristic sequences $(n \mid m)$ and $(n-1,1 \mid m)$ respectively. We should take into account that the superalgebras in [7] show that all null-filiform superalgebras have nilindex $n+m$ (except for a unique superalgebra of maximal nilindex) and there exist filiform superalgebras which also have nilindex $n+m$ for some $n, m$. In the present paper we investigate Leibniz superalgebras with the characteristic sequence $C(L)=(n \mid m-1,1)$ and with nilindex equal to $n+m$. 
In this paper all spaces and superalgebras are considered over the complex number field.

\section{Preliminaries}

We recall the definition of Leibniz superalgebras.

Definition 2.1. A $\mathbb{Z}_{2}$-graded vector space $L=L_{0} \oplus L_{1}$ is called a Leibniz superalgebra if it is equipped with a product $[-,-]$ which satisfies the following conditions:

$$
\begin{aligned}
& {\left[L_{\alpha}, L_{\beta}\right] \subseteq L_{\alpha+\beta(\bmod 2)} \text { for all } \alpha, \beta \in \mathbb{Z}_{2},} \\
& {[x,[y, z]]=[[x, y], z]-(-1)^{\alpha \beta}[[x, z], y] \text { - graded Leibniz identity }}
\end{aligned}
$$

for all $x \in L, y \in L_{\alpha}, z \in L_{\beta}, \alpha, \beta \in \mathbb{Z}_{2}$.

Note that if in $L$ the identity $[x, y]=-(-1)^{\alpha \beta}[y, x]$ (where $x \in L_{\alpha}, y \in L_{\beta}$ )holds, then the graded Leibniz and graded Jacobi identities coincide. Thus, Leibniz superalgebras are a generalization of Lie superalgebras.

Let us anote an example of non Lie Leibniz superalgebras, which generalize the construction of non Lie Leibniz algebras [11].

Let $A=A_{0} \oplus A_{1}$ be an associative superalgebra over a field $F$ and $D: A \rightarrow A$ be a $F$-linear map satisfying the condition:

$$
D(a(D b))=D a D b=D((D a) b)
$$

for all $a, b \in A$. If in the vector space $A$ we define the new product:

$$
\langle a, b\rangle_{D}:=a(D b)-(-1)^{\alpha \beta} D(b) a
$$

for $a \in A_{\alpha}, b \in A_{\beta}, A$ becomes a Leibniz superalgebra.

Let us introduce some notations

$$
\begin{gathered}
\Re(L)=\left\{R_{x} \mid x \in L\right\}, \\
L e i b^{n, m}=\left\{L=L_{0} \oplus L_{1} \mid \operatorname{dim} L_{0}=n, \operatorname{dim} L_{1}=m\right\} .
\end{gathered}
$$

It is not difficult to see that the set $\Re(L)$ will be a Lie superalgebra with the following multiplication:

$$
\left\langle R_{a}, R_{b}\right\rangle:=R_{a} R_{b}-(-1)^{\alpha \beta} R_{b} R_{a}
$$

for all $R_{a} \in \Re(L)_{\alpha}, R_{b} \in \Re(L)_{\beta}$.

Let $V=V_{0} \oplus V_{1}, W=W_{0} \oplus W_{1}$ be two $\mathbb{Z}_{2}$-graded spaces. We say that a linear map $f: V \rightarrow W$ has degree $\alpha$ (denoted as $\operatorname{deg}(f)=\alpha$ ), if $f\left(V_{\beta}\right) \subseteq W_{\alpha+\beta}$ for all $\beta \in \mathbb{Z}_{2}$.

Definition 2.2. Let $L$ and $L^{\prime}$ be Leibniz superalgebras. A linear map $f: L \rightarrow L^{\prime}$ is called a homomorphism of Leibniz superalgebras if

1. $f$ preserves the grading, i.e. $f\left(L_{0}\right) \subseteq L_{0}^{\prime}$ and $f\left(L_{1}\right) \subseteq L_{1}^{\prime}(\operatorname{deg}(f)=0)$;

2. $f([x, y])=[f(x), f(y)]$ for all $x, y \in L$.

Moreover, if $f$ is one-to-one then it is called an isomorphism of Leibniz superalgebras $L$ and $L^{\prime}$. 
For a given Leibniz superalgebra $L$ we define a descending central sequence as follows:

$$
L^{1}=L, \quad L^{k+1}=\left[L^{k}, L^{1}\right], k \geq 1 .
$$

Definition 2.3. A Leibniz superalgebra $L$ is called nilpotent, if there exists $s \in \mathbb{N}$ such that $L^{s}=0$. The minimal number $s$ with this property is called index of nilpotency (nilindex) of the superalgebra $L$.

Definition 2.4. The set $R(L)=\{z \in L \mid[L, z]=0\}$ is called the right annihilator of a superalgebra $L$.

Using the Leibniz graded identity it is not difficult to see that $R(L)$ is an ideal of the superalgebra $L$. Moreover, elements of the form $[a, b]+(-1)^{\alpha \beta}[b, a]\left(a \in L_{\alpha}, b \in L_{\beta}\right)$ belong to $R(L)$.

The description of Leibniz superalgebras of maximal nilindex is represented in the following theorem.

Theorem 2.1. 1]. Let $L$ be an $n$-dimensional Leibniz superalgebra with maximal index of nilpotency. Then $L$ is isomorphic to one of the following two non isomorphic superalgebras:

$$
\begin{gathered}
{\left[e_{i}, e_{1}\right]=e_{i+1}, \quad 1 \leq i \leq n-1} \\
\left\{\begin{array}{l}
{\left[e_{i}, e_{1}\right]=e_{i+1}, \quad 1 \leq i \leq n-1} \\
{\left[e_{i}, e_{2}\right]=2 e_{i+2}, \quad 1 \leq i \leq n-2}
\end{array}\right.
\end{gathered}
$$

where the omitted products are zero.

It should be noted that for the second superalgebra when $n+m$ is even, we have $m=n$ and if $n+m$ is odd then $m=n+1$. Moreover, it is clear that the Leibniz superalgebra has the maximal nilindex if and only if it is one-generated.

We define the characteristic sequence as in [5].

Let $L=L_{0} \oplus L_{1}$ be a nilpotent Leibniz superalgebra. For an arbitrary element $x \in L_{0}$, the operator of right multiplication $R_{x}$ is a nilpotent endomorphism of the space $L_{i}$, where $i \in\{0,1\}$. Let us denote by $C_{i}(x)(i \in\{0,1\})$ the descending sequence of the dimensions of Jordan blocks of the operator $R_{x}$. Consider the lexicographical order on the set $C_{i}\left(L_{0}\right)$.

Definition 2.5. A sequence

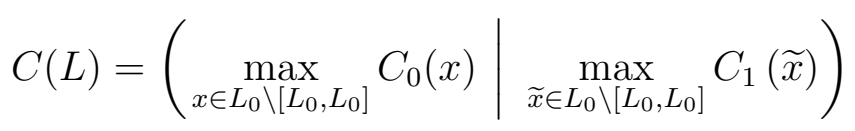

is said to be the characteristic sequence of the Leibniz superalgebra $L$.

Similarly to [6] (corollary 3.0.1) it can be proved that the characteristic sequence is invariant under isomorphisms. 


\section{Description of Leibniz superalgebras with charac- teristic sequence $(n \mid m-1,1)$ and nilindex $n+m$}

The following theorem gives us the location of the generators of the Leibniz superalgebra from Leib $b^{n, m}$ with characteristic sequence $(n \mid m-1,1)$ and nilindex $n+m$.

Theorem 3.1. Let $L=L_{0} \oplus L_{1}$ be a Leibniz superalgebra from the variety Leib ${ }^{n, m}$ with characteristic sequence $(n \mid m-1,1)$ and nilindex $n+m$. Then $L$ is two-generated and they belong to $L_{1}$.

Proof. Since the nilindex of $L$ is $n+m$, then superalgebra $L$ is two-generated. From the definition of characteristic sequence we can conclude that there exists a basis $\left\{y_{1}, y_{2}, \ldots, y_{m}\right\}$ of $L_{1}$ such that the operator $R_{x_{1 \mid L_{1}}}$ in this basis has one of the following forms:

$$
\left(\begin{array}{cc}
J_{n-1} & 0 \\
0 & J_{1}
\end{array}\right), \quad\left(\begin{array}{cc}
J_{1} & 0 \\
0 & J_{n-1}
\end{array}\right)
$$

By a change of the basis elements $\left\{y_{1}, y_{2}, \ldots, y_{m}\right\}$, we can assume that the operator $R_{x_{1 \mid L_{1}}}$ has the first form.

Since $C\left(L_{0}\right)=(n)$, then from [2] (example 1) we have that $L_{0}$ is a zero-filiform Leibniz algebra. Without loss of generality we may suppose that

$$
\begin{cases}{\left[x_{i}, x_{1}\right]=x_{i+1},} & 1 \leq i \leq n-1 \\ {\left[y_{j}, x_{1}\right]=y_{j+1},} & 1 \leq j \leq m-2 \\ {\left[y_{m}, x_{1}\right]=\left[y_{m}, x_{1}\right]=0,} & \end{cases}
$$

where $\left\{x_{1}, x_{2}, \ldots, x_{n}, y_{1}, y_{2}, \ldots, y_{m}\right\}$ is the basis of the superalgebra $L$.

From these multiplications we deduce that $\left\{x_{2}, x_{3}, \ldots, x_{n}\right\}$ lie in the right annihilator of the superalgebra $L$ and generators can be selected as a linear combination of elements of the set $\left\{x_{1}, y_{1}, y_{m}\right\}$.

It is clear that two generators can not lie in $L_{0}$. Let us suppose the opposite assertion to the assertion of the theorem, i.e. one generator element lies in $L_{0}$ and the second lies in $L_{1}$. Then we can choose as generators the elements $x_{1}, A y_{1}+B y_{m}$.

Case 1. Let us suppose $A=0$. Then $x_{1}$ and $y_{m}$ can be chosen as generators. Hence

$$
L^{2}=\left\{x_{2}, x_{3}, \ldots, x_{n}, y_{1}, y_{2}, \ldots, y_{m-1}\right\} .
$$

Introduce the notations

$$
\left[y_{1}, y_{m}\right]=\sum_{k=2}^{n} \beta_{k} x_{k}, \quad\left[x_{1}, y_{m}\right]=\sum_{s=1}^{m-1} \alpha_{s} y_{s} .
$$

Consider the products

$$
\left[x_{i},\left[y_{m}, x_{1}\right]\right]=\left[\left[x_{i}, y_{m}\right], x_{1}\right]-\left[\left[x_{i}, x_{1}\right], y_{m}\right], 1 \leq i \leq n .
$$

On the other hand, $\left[x_{i},\left[y_{m}, x_{1}\right]\right]=0$. 
Therefore

$$
\begin{array}{ll}
{\left[x_{i}, y_{m}\right]=\sum_{k=1}^{m-i} \alpha_{k} y_{k-1+i},} & 1 \leq i \leq \min \{n, m-1\} \\
{\left[x_{i}, y_{m}\right]=0,} & \min \{n, m-1\}<i \leq n
\end{array}
$$

Thus, we obtain $y_{1} \notin\left\langle\left[x_{i}, y_{m}\right]\right\rangle, 2 \leq i \leq n$ and since $y_{1} \in L^{2}$, then $\alpha_{1} \neq 0$. Consider the products

$$
\left[y_{j},\left[y_{m}, x_{1}\right]\right]=\left[\left[y_{j}, y_{m}\right], x_{1}\right]-\left[\left[y_{j}, x_{1}\right], y_{m}\right], 1 \leq j \leq m-2 .
$$

On the other hand we have $\left[y_{j},\left[y_{m}, x_{1}\right]\right]=0, \quad 1 \leq j \leq m-2$.

Therefore

$$
\begin{array}{ll}
{\left[y_{j}, y_{m}\right]=\sum_{s=2}^{n+1-j} \beta_{s} x_{s-1+j},} & 1 \leq j \leq \min \{m-1, n-1\} \\
{\left[y_{j}, y_{m}\right]=0,} & \min \{n-1, m-1\}<j \leq m-1 .
\end{array}
$$

Consider the equalities

$$
\left[x_{1},\left[y_{m}, y_{m}\right]\right]=2\left[\left[x_{1}, y_{m}\right], y_{m}\right]=2\left[\sum_{s=1}^{m-1} \alpha_{s} y_{s}, y_{m}\right]=\sum_{s=1}^{m-1} \alpha_{s} \sum_{t=2}^{n+1-s} \beta_{t} x_{t-1+s}
$$

Since $\left[y_{m}, y_{m}\right] \in\left\langle x_{2}, x_{3}, \ldots, x_{n}\right\rangle$ then $\left[y_{m}, y_{m}\right] \in R(L)$ and hence $\left[x_{1},\left[y_{m}, y_{m}\right]\right]=0$. Therefore,

$$
\sum_{s=1}^{m-1} \alpha_{s} \sum_{t=2}^{n+1-s} \beta_{t} x_{t-1+s}=0 .
$$

Comparing the coefficient at the basic elements $x_{i}, 2 \leq i \leq n$, we obtain $\beta_{i}=0,2 \leq$ $i \leq n$. And we have $L^{3}=\left\{x_{3}, x_{4}, \ldots, x_{n}, y_{2}, y_{3}, \ldots, y_{m-1}\right\}$, but it follows that the index of nilpotency of the superalgebra $L$ is smaller than $n+m$. Thus, we have contradiction with assumption $A=0$.

Case 2. $A \neq 0$. Then we can take as generators $x_{1}$ and $y_{1}$. Hence, $L^{2}=$ $\left\{x_{2}, x_{3}, \ldots, x_{n}, y_{2}, y_{3}, \ldots, y_{m}\right\}$.

Let us define the notations

$$
\left[x_{i}, y_{1}\right]=\sum_{k=2}^{m} \alpha_{i, k} y_{k}, 1 \leq i \leq n, \quad\left[y_{j}, y_{1}\right]=\sum_{s=2}^{n} \beta_{j, s} x_{s}, 1 \leq j \leq m .
$$

Let us suppose $x_{2} \notin L^{3}$. Then $L^{3}=\left\{x_{3}, x_{4}, \ldots, x_{n}, y_{2}, y_{3}, \ldots, y_{m}\right\}$ and there exist some $i_{0}, j_{0}\left(2 \leq i_{0}, j_{0}\right)$ such that $\alpha_{i_{0}, 2} \alpha_{j_{0}, m}-\alpha_{j_{0}, 2} \alpha_{i_{0}, m} \neq 0$ and $\alpha_{i_{0}, 2} \alpha_{j_{0}, m} \neq 0$.

Since $\left[x_{i_{0}}, y_{1}\right]=\sum_{k=2}^{m} \alpha_{i_{0}, k} y_{k} \in R(L)$ and $\alpha_{i_{0}, 2} \neq 0$ then multiplying from the right side by $x_{1}$ enough times, we obtain $\left\{y_{3}, y_{4}, \ldots, y_{m-1}\right\} \subseteq R(L)$.

Therefore

$$
\left[x_{i_{0}, 2}, y_{1}\right]-\sum_{k=3}^{m-1} \alpha_{i_{0}, k} y_{k}=\alpha_{i_{0}, 2} y_{2}+\alpha_{i_{0}, m} y_{m} \in R(L)
$$




$$
\left[x_{j_{0}, 2}, y_{1}\right]-\sum_{s=3}^{m-1} \alpha_{j_{0}, s} y_{s}=\alpha_{j_{0}, 2} y_{2}+\alpha_{j_{0}, m} y_{m} \in R(L) .
$$

Since $\alpha_{i_{0}, 2} \alpha_{j_{0}, m}-\alpha_{j_{0}, 2} \alpha_{i_{0}, m} \neq 0$, then we have $y_{2}, y_{m} \in R(L)$, i.e. $\left\{y_{2}, y_{3}, \ldots, y_{m}\right\} \subseteq$ $R(L)$ and hence $\left[x_{1}, y_{1}\right] \in R(L)$.

From equality

$$
\left[\left[x_{i-1}, x_{1}\right], y_{1}\right]=\left[x_{i-1},\left[x_{1}, y_{1}\right]\right]+\left[\left[x_{i-1}, y_{1}\right], x_{1}\right]
$$

we obtain

$$
\begin{array}{ll}
{\left[x_{i}, y_{1}\right]=\sum_{k=2}^{m-i} \alpha_{1, k} y_{k+i-1},} & 2 \leq i \leq \min \{n, m-2\} \\
{\left[x_{i}, y_{1}\right]=0,} & \min \{n, m-2\}<i \leq n
\end{array}
$$

from which we have a contradiction to the assumption $\alpha_{i_{0}, 2} \neq 0$.

Therefore $x_{2} \in L^{3}$ and $x_{2}$ is a linear combination of products $\left[y_{i}, y_{1}\right], 1 \leq i \leq m$, hence $\left[x_{2}, y_{1}\right]=\sum_{i=1}^{m} \gamma_{i}\left[\left[y_{i}, y_{1}\right], y_{1}\right]$. Using the Leibniz graded identity and $\left[y_{1}, y_{1}\right] \in R(L)$ one can easy see that $\left[x_{2}, y_{1}\right]=0$.

Thus, we have $L^{3}=\left\{x_{2}, x_{3}, \ldots, x_{n}, C y_{2}+D y_{m}, y_{3}, \ldots, y_{m-1}\right\}$.

Since $\left[x_{2}, y_{1}\right]=0$, then $C y_{2}+D y_{m} \in L^{3}$ should be expressed by linear combinations of products $\left[x_{i}, y_{1}\right], 3 \leq i \leq n$. Therefore, $C y_{2}+D y_{m} \in L^{5}$.

Moreover, if $C \neq 0$, then $C y_{2}+D y_{m} \in R(L)$. Then multiplying from the right side by $x_{1}$ enough times, we obtain $\left\{y_{3}, y_{4}, \ldots, y_{m-1}\right\} \subseteq R(L)$.

Since $\left[x_{2}, y_{1}\right]=0$ and $C y_{2}+D y_{m} \in L^{3}$ then there exist $t_{0}, t_{0} \geq 3$, such that $\left[x_{t_{0}}, y_{1}\right]=c_{2}\left(A y_{2}+B y_{m}\right)+c_{3} y_{3}+\cdots+c_{m-1} y_{m-1}, c_{2} \neq 0$.

Applying the Leibniz graded identity we obtain

$$
\left[x_{1},\left[\ldots\left[[y_{1}, \underbrace{\left.\left.x_{1}\right], x_{1}\right], \ldots, x_{1}}_{t_{0}-1-\text { times }}]\right]=(-1)^{t_{0}-1} c_{2}\left(C y_{2}+D y_{m}\right)+\left\{y_{3}, y_{4}, \ldots, y_{m-1}\right\}\right. \text {. }\right.
$$

As $L$ is nilpotent, we have existence $s \in \mathbb{N}$, such that $y_{t_{0}} \in L^{s} \backslash L^{s+1}$.

If $t_{0}<m$, then $\left[x_{1}, y_{t_{0}}\right]=(-1)^{t_{0}-1} c_{2}\left(C y_{2}+D y_{m}\right)+\left\{y_{3}, y_{4}, \ldots, y_{m-1}\right\} \in L^{s+1}$ and multiplying this equality $\left(t_{0}-2\right)$ times from the right side by $x_{1}$, we obtain that $(-1)^{i_{0}-1} c_{2} C y_{t_{0}} \in L^{s+t_{0}-1}$. The inequality $s+t_{0}-1>s$ contradicts the condition $y_{t_{0}} \in L^{s} \backslash L^{s+1}$ and hence we obtain $C=0$.

If $t_{0} \geq m$, then $\left[x_{1}, y_{t_{0}}\right]=0$. From (3.2) we again obtain $C=0$.

Thus, $L^{3}=\left\{x_{2}, x_{3}, \ldots, x_{n}, y_{3}, \ldots, y_{m-1}, y_{m}\right\}$.

Consider the following subcases.

Case 2.1 Let $\alpha_{1,2} \neq 0$. Suppose that $x_{2} \in L^{l} \backslash L^{l+1}$ for some $l(3 \leq l \leq m)$. Then

$$
\begin{aligned}
L^{l} & =\left\{x_{2}, x_{3}, \ldots, x_{n}, y_{l}, \ldots, y_{m-1}, y_{m}\right\} . \\
L^{l+1} & =\left\{x_{3}, x_{4}, \ldots, x_{n}, y_{l}, \ldots, y_{m-1}, y_{m}\right\} .
\end{aligned}
$$


Since $x^{2} \in L^{l} \backslash L^{l+1}$, we obtain $\beta_{l-1,2} \neq 0$, i.e.

$$
\left[y_{l-1}, y_{1}\right]=\sum_{k=2}^{n} \beta_{l-1, k} x_{k} .
$$

The equality $\left[x_{2}, y_{1}\right]=0$, deduce $y_{l} \in L^{l+2}$ and $L^{l+2}=\left\{x_{4}, x_{5}, \ldots, x_{n}, y_{l}, \ldots, y_{m-1}, y_{m}\right\}$.

Therefore $\left[y_{l}, y_{1}\right]=\sum_{k=4}^{n} \beta_{l, k} x_{k}$, i.e. $\beta_{l, 2}=\beta_{l, 3}=0$

In notation (3.1) by induction on $j$ for any value of $i$ one can prove the following equality:

$$
\left[y_{i}, y_{j}\right]=\sum_{k=0}^{\min \{i+j-1, m-1\}-i}(-1)^{k} C_{j-1}^{k} \sum_{t=2}^{n-j+k+1} \beta_{i+k, t} x_{t+j-k-1},
$$

where $1 \leq j \leq m-1,1 \leq i \leq m-1$.

From (3.3) we have $\left[y_{2}, y_{l}\right]=\beta_{l-1,2} x_{3}+x_{4}, x_{5}, \ldots, x_{n}$

Consider the equalities

$$
\begin{gathered}
{\left[x_{1},\left[y_{1}, y_{l}\right]\right]=\left[\left[x_{1}, y_{1}\right], y_{l}\right]+\left[\left[x_{1}, y_{l}\right], y_{1}\right]=} \\
=\sum_{k=2}^{m} \alpha_{1, k}\left[y_{k}, y_{l}\right]+\sum_{s=l+1}^{m} \gamma_{1, s}\left[y_{k}, y_{1}\right]=\alpha_{1,2} \beta_{l-1,2} x_{3}+\left\{x_{4}, \ldots, x_{n}\right\} .
\end{gathered}
$$

On the other hand

$$
\left[x_{1},\left[y_{1}, y_{l}\right]\right]=0 \text {. }
$$

So $\alpha_{1,2} \beta_{l-1,2} x_{3}=0$, i.e. we have a contradiction with supposition $\alpha_{1,2} \neq 0$.

Case 2.1. If $\alpha_{1,2}=0$. Then $\left[y_{1}, x_{1}\right]+\left[x_{1}, y_{1}\right]=y_{2}+\alpha_{1,3} y_{3}+\alpha_{1,4} y_{4}+\cdots+$ $\alpha_{1, m} y_{m} \in R(L)$ and then multiplying from the right side by $x_{1}$ enough times, we obtain $y_{2}+\alpha_{1, m} y_{m}, y_{3}, \ldots, y_{m-1} \in R(L)$.

Since $y_{m} \in L^{3}$, there exists $i_{0} \geq 2$ such that

$\left[x_{i_{0}}, y_{1}\right]=\alpha_{i_{0}, 3} y_{3}+\alpha_{i_{0}, 4} y_{4}+\cdots+\alpha_{i_{0}, m} y_{m}, \alpha_{i_{0}, m} \neq 0$.

From $\left[x_{i_{0}}, y_{1}\right] \in R(L)$, we have $\alpha_{i_{0}, 3} y_{3}+\alpha_{i_{0}, 4} y_{4}+\cdots+\alpha_{i_{0}, m} y_{m}, \in R(L)$. Therefore $y_{2}, y_{m} \in R(L)$.

Consider the equalities

$$
\begin{aligned}
& {\left[y_{j},\left[y_{1}, x_{1}\right]\right]=\left[\left[y_{j}, y_{1}\right], x_{1}\right]-\left[\left[y_{j}, x_{1}\right], y_{1}\right]=\left[\sum_{k=2}^{n+1-j} \beta_{j, k} x_{k-1+j}, x_{1}\right]-\left[y_{j+1}, y_{1}\right]=} \\
& =\sum_{k=2}^{n-j} \beta_{j, k} x_{k+j}-\left[y_{j+1}, y_{1}\right] .
\end{aligned}
$$

On the other hand we have $\left[y_{j},\left[y_{1}, x_{1}\right]\right]=\left[y_{j}, y_{2}\right]=0$.

Therefore

$$
\begin{aligned}
& {\left[y_{j+1}, y_{1}\right]=\sum_{k=2}^{n-j} \beta_{1,2} x_{k+j}, 1 \leq j \leq \min \{n-2, m-2\}} \\
& {\left[y_{j+1}, y_{1}\right]=0 \min \{n-2, m-2\} \leq j \leq m-2 .}
\end{aligned}
$$

From these we conclude that $x_{2}$ cannot be expressed via linear combination of $\left\{\left[y_{j}, y_{1}\right]\right\}, 2 \leq j \leq m$. Therefore $x_{2} \notin L^{3}$, it is a contradiction with assumption $x_{2} \in L^{3}$.

Thus, the supposition of the case when one generator lies in $L_{0}$ and the second lies in $L_{1}$ leads to a contradiction, therefore both generators belong to $L_{1}$ and the proof of the theorem is completed. 
The following lemma is an important technical result in our description.

Lemma 3.1. Let $L=L_{0} \oplus L_{1}$ be a Leibniz superalgebra from the variety Leib ${ }^{n, m}$ with characteristic sequence $(n \mid m-1,1)$ and nilindex $n+m$. Then there exists $y \in L_{1}$ such that the superalgebra $\langle y\rangle$ is isomorphic to the superalgebra from Theorem [2.1 and $m \in\{n+1, n+2\}$.

Proof. From Theorem 3.1 we have that both generators are in $L_{1}$. Let $\left\{x_{1}, x_{2}, \ldots, x_{n}, y_{1}, y_{2}, \ldots, y_{m}\right\}$ be the basis of $L$ such that $y_{1}, y_{m}$ are the generators and

$$
\begin{gathered}
{\left[x_{i}, x_{1}\right]=x_{i+1}, 1 \leq i \leq n-1,\left[y_{j}, x_{1}\right]=y_{j+1}, 1 \leq j \leq m-2,} \\
{\left[y_{1}, y_{1}\right]=\sum_{i=1}^{n} a_{i} x_{i},\left[y_{m}, y_{1}\right]=\sum_{i=1}^{n} b_{i} x_{i},} \\
{\left[y_{1}, y_{m}\right]=\sum_{i=1}^{n} c_{i} x_{i},\left[y_{m}, y_{m}\right]=\sum_{i=1}^{n} d_{i} x_{i} .}
\end{gathered}
$$

Since $\left[y_{j}, x_{1}\right]=y_{j+1}, 1 \leq j \leq m-2$, then $x_{1}$ cannot be generated via multiplications of the elements $\left\{y_{2}, y_{3}, \ldots, y_{m-1}\right\}$. Therefore $x_{1} \in\left\langle\left[y_{1}, y_{1}\right],\left[y_{m}, y_{1}\right],\left[y_{1}, y_{m}\right],\left[y_{m}, y_{m}\right]\right\rangle$ and hence $\left(a_{1}, b_{1}, c_{1}, d_{1} \neq(0,0,0,0)\right.$.

Let us suppose that $a_{1} \neq 0$. Then making the change

$$
x_{i}^{\prime}=\sum_{k=1}^{n+1-i} a_{k} x_{k+i-1}, 1 \leq i \leq n, y_{j}^{\prime}=a_{1}^{i-1} y_{j}, 1 \leq j \leq m-1
$$

we obtain

$$
\left[x_{i}, x_{1}\right]=x_{i+1}, 1 \leq i \leq n-1,\left[y_{j}, x_{1}\right]=y_{j+1}, 1 \leq j \leq m-2,\left[y_{1}, y_{1}\right]=x_{1} .
$$

It is clear that the subsuperalgebra $\left\langle y_{1}\right\rangle=\left\{x_{1}, x_{2}, \ldots, x_{n}, y_{1}, y_{2}, \ldots, y_{m-1}\right\}$ is onegenerated and therefore it has maximal index of nilpotency. Hence from Theorem 2.1 we have that either $m=n+1$ or $m=n+2$.

Let us suppose now that $a_{1}=0$.

Consider the product

$$
\left[y_{m},\left[y_{m}, x_{1}\right]\right]=\left[\left[y_{m}, y_{m}\right], x_{1}\right]-\left[\left[y_{m}, x_{1}\right], y_{m}\right]
$$

Then $\left[y_{m}, y_{m}\right]=d_{n} x_{n}$ and $d_{i}=0,1 \leq i \leq n-1$. It leads to $\left(b_{1}, c_{1}\right) \neq(0,0)$.

From $\left[y_{m}, y_{1}\right]-\left[y_{1}, y_{m}\right]=\sum_{i=1}^{n}\left(b_{i}-c_{i}\right) x_{i} \in R(L)$ and $x_{j} \in R(L), 2 \leq j \leq n$ we obtain that $\left(b_{1}-c_{1}\right) x_{1} \in R(L)$, but $x_{1} \notin R(L)$ and hence $b_{1}=c_{1} \neq 0$.

By the following change of basis:

$$
x_{1}^{\prime}=\sum_{k=1}^{n} b_{k} x_{k}, x_{i+1}^{\prime}=\left[x_{i}^{\prime}, x_{1}^{\prime}\right], 1 \leq i \leq n-1, y_{j}^{\prime}=y_{j}, \quad 1 \leq j \leq m
$$

we can assume $\left[y_{m}, y_{1}\right]=x_{1}$. 
From the products

$$
\begin{gathered}
{\left[y_{m},\left[y_{i}, x_{1}\right]\right]=\left[\left[y_{m}, y_{i}\right], x_{1}\right]-\left[\left[y_{m}, x_{1}\right], y_{i}\right],} \\
{\left[y_{m},\left[y_{1}, y_{1}\right]\right]=2\left[\left[y_{m}, y_{1}\right], y_{1}\right]=2\left[x_{1}, y_{1}\right]}
\end{gathered}
$$

we obtain $\left[y_{m}, y_{i+1}\right]=x_{i+1}, 1 \leq i \leq m-2,\left[x_{1}, y_{1}\right]=0$.

Since $\left[y_{1}, x_{1}\right]+\left[x_{1}, y_{1}\right] \in R(L)$ then $y_{2} \in R(L)$, but this contradicts $\left[y_{m}, y_{2}\right]=x_{2}$ and therefore the case $a_{1}=0$ is not possible either.

The existence of an adapted basis with conditions of Lemma 3.1 in case of $m=n+1$ is described in the following lemma.

Lemma 3.2. Let $L$ be a Leibniz superalgebra from the variety Leib ${ }^{n, m}$ with characteristic sequence $(n \mid m-1,1)$ and nilindex $n+m$. Then, in case $m=n+1$, there exists a basis $\left\{x_{1}, x_{2}, \ldots, x_{n}, y_{1}, y_{2}, \ldots, y_{n+1}\right\}$ of $L$ in which the products have the following form:

$$
\begin{array}{ll}
{\left[x_{i}, x_{1}\right]=x_{i+1}, 1 \leq i \leq n-1,} & {\left[y_{j}, x_{1}\right]=y_{j+1}, 1 \leq j \leq n-1,} \\
{\left[x_{i}, y_{1}\right]=\frac{1}{2} y_{i+1}, 1 \leq i \leq n-1,} & {\left[y_{j}, y_{1}\right]=x_{j}, 1 \leq j \leq n,} \\
{\left[y_{n+1}, y_{n+1}\right]=\gamma x_{n},} & {\left[x_{i}, y_{n+1}\right]=\sum_{k=\left[\frac{n+4}{2}\right]}^{n+1-i} \beta_{k} y_{k-1+i}, 1 \leq i \leq\left[\frac{n-1}{2}\right],} \\
{\left[y_{1}, y_{n+1}\right]=-2 \sum_{k=\left[\frac{n+4}{2}\right]}^{n+2-j} \beta_{k} x_{k-1}+\beta x_{n},} & {\left[y_{j}, y_{n+1}\right]=-2 \sum_{k=\left[\frac{n+4}{2}\right]}^{n+2} \beta_{k} x_{k-2+j}, 2 \leq j \leq\left[\frac{n+1}{2}\right] .}
\end{array}
$$

Proof. From Lemma 3.1 and Theorem 2.1 we have that there exists a basis $\left\{x_{1}, x_{2}, \ldots, x_{n}, y_{1}, y_{2}, \ldots, y_{n+1}\right\}$ in which products have the following form:

$$
\begin{array}{ll}
{\left[x_{i}, x_{1}\right]=x_{i+1}, 1 \leq i \leq n-1,} & {\left[y_{j}, x_{1}\right]=y_{j+1}, 1 \leq j \leq n-1,} \\
{\left[x_{i}, y_{1}\right]=\frac{1}{2} y_{i+1}, 1 \leq i \leq n-1,} & {\left[y_{j}, y_{1}\right]=x_{j}, 1 \leq j \leq n,} \\
{\left[y_{1}, y_{n+1}\right]=\sum_{k=1}^{n} \alpha_{k} x_{k},} & {\left[x_{1}, y_{n+1}\right]=\sum_{s=2}^{n} \beta_{s} y_{s} .}
\end{array}
$$

From equalities:

$$
\begin{gathered}
{\left[y_{n+1},\left[y_{1}, x_{1}\right]\right]=\left[\left[y_{n+1}, y_{1}\right], x_{1}\right]-\left[\left[y_{n+1}, x_{1}\right], y_{1}\right],} \\
{\left[y_{n+1},\left[x_{1}, y_{1}\right]\right]=\left[\left[y_{n+1}, x_{1}\right], y_{1}\right]-\left[\left[y_{n+1}, y_{1}\right], x_{1}\right],} \\
{\left[y_{n+1}, y_{i+1}\right]=\left[y_{n+1},\left[y_{i}, x_{1}\right]\right]=\left[\left[y_{n+1}, y_{i}\right], x_{1}\right]-\left[\left[y_{n+1}, x_{1}\right], y_{i}\right], 1 \leq i \leq n-1,}
\end{gathered}
$$

we have $\left[y_{n+1}, y_{2}\right]=\left[\left[y_{n+1}, y_{1}\right], x_{1}\right]$ and $\left[y_{n+1}, y_{2}\right]=-2\left[\left[y_{n+1}, y_{1}\right], x_{1}\right]$, it follows that

$\left[y_{n+1}, y_{1}\right]=b x_{n}$ and $\left[y_{n+1}, y_{i}\right]=0$ for $2 \leq i \leq n$.

Consider the equality:

$$
\left[y_{n+1},\left[y_{n+1}, x_{1}\right]\right]=\left[\left[y_{n+1}, y_{n+1}\right], x_{1}\right]+\left[\left[y_{n+1}, x_{1}\right], y_{n+1}\right],
$$

then $\left[y_{n+1}, y_{n+1}\right]=\gamma x_{n}$.

Considering the graded identities:

$$
\left[y_{j},\left[y_{n+1}, x_{1}\right]\right]=\left[\left[y_{j}, y_{n+1}\right], x_{1}\right]-\left[\left[y_{j}, x_{1}\right], y_{n+1}\right]
$$




$$
\left[x_{i},\left[y_{n+1}\right], x_{1}\right]=\left[\left[x_{i}, y_{n+1}\right], x_{1}\right]-\left[\left[x_{i}, x_{1}\right], y_{n+1}\right]
$$

we have

$$
\begin{gathered}
{\left[y_{j}, y_{n+1}\right]=\sum_{k=1}^{n+1-j} \alpha_{k} x_{k-1+j}, 2 \leq j \leq n,} \\
{\left[x_{i}, y_{n+1}\right]=\sum_{s=2}^{n+1-i} \beta_{s} y_{s-1+i}, 2 \leq i \leq n-1 .}
\end{gathered}
$$

From the chain of equalities

$$
\begin{aligned}
& 0=\left[y_{1},\left[y_{n+1}, y_{1}\right]\right]=\left[\left[y_{1}, y_{n+1}\right], y_{1}\right]+\left[\left[y_{1}, y_{1}\right], y_{n+1}\right]= \\
& =\left[\sum_{k=1}^{n} \alpha_{k} x_{k}, y_{1}\right]+\left[x_{1}, y_{n+1}\right]=\frac{1}{2} \sum_{k=1}^{n-1} \alpha_{k} y_{k+1}+\sum_{s=2}^{n} \beta_{s} y_{s},
\end{aligned}
$$

we obtain $\alpha_{i}=-2 \beta_{i+1}, 1 \leq i \leq n-1$.

Substituting these relations in the chain of equalities

$$
0=\left[y_{1},\left[y_{n+1}, y_{n+1}\right]\right]=2\left[\left[y_{1}, y_{n+1}\right], y_{n+1}\right],
$$

we get $\beta_{i}=0,2 \leq i \leq\left[\frac{n+2}{2}\right]$. Thus, we obtain the multiplications of the superalgebra as in the assertion of the lemma.

For convenience, we will denote the superalgebra from the family of Lemma 3.2 as $L\left(\gamma, \beta_{\left[\frac{n+4}{2}\right]}, \beta_{\left[\frac{n+4}{2}\right]+1}, \ldots, \beta_{n}, \beta\right)$.

Using the properties of adapted basis we obtain necessary and sufficient conditions when two arbitrary superalgebras from the family of Lemma 3.2 are isomorphic.

Theorem 3.2. Two superalgebras $L\left(\gamma, \beta_{\left[\frac{n+4}{2}\right]}, \beta_{\left[\frac{n+4}{2}\right]+1}, \ldots, \beta_{n}, \beta\right)$ and

$L^{\prime}\left(\gamma^{\prime}, \beta_{\left[\frac{n+4}{2}\right]}^{\prime}, \beta_{\left[\frac{n+4}{2}\right]+1}^{\prime}, \ldots, \beta_{n}^{\prime}, \beta^{\prime}\right)$ are isomorphic if and only if there exist $a_{1}, a_{n+1}, b_{n+1} \in$ $\mathbb{C}$ such that the following conditions hold:

for odd $n$ :

$$
\left\{\begin{array}{l}
b_{n+1}^{2} \gamma=\gamma^{\prime} a_{1}^{2 n} \\
b_{n+1} \beta_{j}=a_{1}^{2 j-3} \beta_{j}^{\prime}, \quad\left[\frac{n+4}{2}\right] \leq j \leq n \\
a_{n+1} b_{n+1} \gamma+a_{1} b_{n+1} \beta=a_{1}^{2 n} \beta^{\prime}+4 \beta_{\left[\frac{n+4}{2}\right]}^{\prime} a_{1}^{2\left[\frac{n+1}{2}\right]-1} a_{n+1} \beta_{\left[\frac{n+4}{2}\right]}
\end{array}\right.
$$

for even $n$ :

$$
\left\{\begin{array}{l}
b_{n+1}^{2} \gamma=\gamma^{\prime} a_{1}^{2 n} \\
b_{n+1} \beta_{j}=a_{1}^{2 j-3} \beta_{j}^{\prime}, \quad\left[\frac{n+4}{2}\right] \leq j \leq n \\
a_{n+1} b_{n+1} \gamma+a_{1} b_{n+1} \beta=a_{1}^{2 n} \beta^{\prime}
\end{array}\right.
$$


Proof. Let us make a general change of generator elements in the form:

$$
y_{1}^{\prime}=\sum_{i=1}^{n+1} a_{i} y_{i}, y_{n+1}^{\prime}=\sum_{j=1}^{n+1} b_{j} y_{j}
$$

where the $\operatorname{rank}\left(\begin{array}{cccc}a_{1} & a_{2} & \ldots & a_{n+1} \\ b_{1} & b_{2} & \ldots & b_{n+1}\end{array}\right)=2$.

We express the new basis $\left\{x_{1}^{\prime}, x_{2}^{\prime}, \ldots, x_{n}^{\prime}, y_{1}^{\prime}, y_{2}^{\prime}, \ldots, y_{n+1}^{\prime}\right\}$ of the superalgebra $L^{\prime}\left(\gamma^{\prime}, \beta_{\left[\frac{n+4}{2}\right]}^{\prime}, \beta_{\left[\frac{n+4}{2}\right]+1}^{\prime}, \ldots, \beta_{n}^{\prime}, \beta^{\prime}\right)$ with respect to the old basis $\left\{x_{1}, x_{2}, \ldots, x_{n}, y_{1}, y_{2}\right.$, $\left.\ldots, y_{n+1}\right\}$.

Then for element $x_{1}^{\prime}$ we have

$$
\begin{gathered}
x_{1}^{\prime}=\left[y_{1}^{\prime}, y_{1}^{\prime}\right]=a_{1} \sum_{i=1}^{n} a_{i} x_{i}-2 a_{1} a_{n+1} \sum_{k=\left[\frac{n+4}{2}\right]}^{n} \beta_{k} x_{k-1}+ \\
+a_{1} a_{n+1} \beta x_{n}-2 a_{n+1} \sum_{i=2}^{\left[\frac{n+1}{2}\right]} a_{i} \sum_{k=\left[\frac{n+4}{2}\right]}^{n+2-i} \beta_{k} x_{k+i-2}+a_{n+1}^{2} \gamma x_{n} .
\end{gathered}
$$

The expression of $x_{t+1}^{\prime}\left(1 \leq t \leq\left[\frac{n-1}{2}\right]\right)$ will be as follows:

$$
x_{t+1}^{\prime}=\left[x_{t}^{\prime}, x_{1}^{\prime}\right]=a_{1}^{2 t+1} \sum_{i=1}^{n-t} a_{i} x_{i+t}-2 a_{1}^{2 t} a_{n+1} \sum_{i=1}^{\left[\frac{n+1}{2}\right]-t} a_{i} \sum_{k=\left[\frac{n+4}{2}\right]}^{n+2-t-i} \beta_{k} x_{k+t+i-2} .
$$

And for $x_{t+1}^{\prime}\left(\left[\frac{n+1}{2}\right] \leq t \leq n-1\right)$ we have

$$
x_{t+1}^{\prime}=\left[x_{t}^{\prime}, x_{1}^{\prime}\right]=a_{1}^{2 t+1} \sum_{i=1}^{n-t} a_{i} x_{t+i} .
$$

For basis elements $y_{i}^{\prime}$ of the space $L_{1}^{\prime}$ basis we have:

$$
y_{t}^{\prime}=\left[y_{t-1}^{\prime}, x_{1}^{\prime}\right]=a_{1}^{2(t-1)} \sum_{j=1}^{n+1-t} a_{i} y_{t+j-1}, 2 \leq t \leq n .
$$

Consider the equalities:

$$
\begin{gathered}
{\left[y_{n+1}^{\prime}, y_{n+1}^{\prime}\right]=b_{1} \sum_{i=1}^{n} b_{i} x_{i}-2 b_{1} b_{n+1} \sum_{k=\left[\frac{n+4}{2}\right]}^{n} \beta_{k} x_{k-1}+b_{1} b_{n+1} \beta x_{n}-} \\
-2 b_{n+1} \sum_{i=2}^{\left[\frac{n+1}{2}\right]} b_{i} \sum_{k=\left[\frac{n+4}{2}\right]}^{n+2-i} \beta_{k} x_{k+i-2}+b_{n+1}^{2} \gamma x_{n}=\gamma^{\prime} x_{n}^{\prime}=\gamma^{\prime} a_{1}^{2 n} x_{n} .
\end{gathered}
$$


Comparing the coefficients we obtain the following restrictions:

$$
\begin{gathered}
b_{1}=b_{2}=\ldots=b_{\left[\frac{n-1}{2}\right]}=0 \\
-2 b_{n+1} b_{\left[\frac{n+1}{2}\right]} \beta_{\left[\frac{n+4}{2}\right]}+b_{n+1}^{2} \gamma=\gamma^{\prime} a_{1}^{2 n} .
\end{gathered}
$$

Using the chain of the equalities:

$$
\left[y_{n+1}^{\prime}, y_{1}^{\prime}\right]=a_{1} \sum_{i=\left[\frac{n+1}{2}\right]}^{n} b_{i} x_{i}+a_{n+1} b_{n+1} \gamma x_{n}-2 a_{n+1} b_{\left[\frac{n+1}{2}\right]} \beta_{\left[\frac{n+4}{2}\right]} x_{n}=0
$$

the equalities (3.4) - (3.5) and comparing the coefficients to the basis elements we obtain:

$$
\left\{\begin{array}{l}
b_{1}=b_{2}=b_{\left[\frac{n-1}{2}\right]}=b_{\left[\frac{n+1}{2}\right]}=\ldots=b_{n-1}=0 \\
b_{n+1}^{2} \gamma=\gamma^{\prime} a_{1}^{2 n} \\
a_{1} b_{n}+a_{n+1} b_{n+1} \gamma=0
\end{array}\right.
$$

Therefore, $y_{n+1}^{\prime}=b_{n} y_{n}+b_{n+1} y_{n+1}$.

Consider the products:

$$
\begin{gathered}
{\left[x_{1}^{\prime}, y_{n+1}^{\prime}\right]=\left[a_{1} \sum_{i=1}^{n} a_{i} x_{i}-2 a_{1} a_{n+1} \sum_{k=\left[\frac{n+4}{2}\right]}^{n} \beta_{k} x_{k-1}+a_{1} a_{n+1} \beta x_{n}-\right.} \\
\left.-2 a_{n+1} \sum_{i=2}^{\left[\frac{n+1}{2}\right]} a_{i} \sum_{k=\left[\frac{n+4}{2}\right]}^{n+2-i} \beta_{k} x_{k+i-2}+a_{n+1}^{2} \gamma x_{n}, b_{n} y_{n}+b_{n+1} y_{n+1}\right]= \\
=a_{1} b_{n+1} \sum_{i=1}^{\left[\frac{n-1}{2}\right]} a_{i} \sum_{k=\left[\frac{n+4}{2}\right]}^{n+1-i} \beta_{k} y_{k+i-1}, \\
\left.\sum_{1}^{\prime}, y_{n+1}^{\prime}\right]=\beta_{k=\left[\frac{n+4}{2}\right]}^{n} \beta_{k}^{\prime} y_{k}^{\prime}=\beta_{\left[\frac{n+4}{2}\right]}^{\prime 2\left[\frac{n+4}{2}\right]-2}\left(a_{1} y_{\left[\frac{n+4}{2}\right]}+a_{2} y_{\left[\frac{n+4}{2}\right]+1}+\ldots+a_{n+1-\left[\frac{n+4}{2}\right]} y_{n}\right)+ \\
+\beta_{\left[\frac{n+4}{2}\right]+1}^{\prime} a_{1}^{2\left[\frac{n+4}{2}\right]}\left(a_{1} y_{\left[\frac{n+4}{2}\right]+1}+a_{2} y_{\left[\frac{n+4}{2}\right]+2}+\ldots+a_{n-\left[\frac{n+4}{2}\right]} y_{n}\right)+\ldots+ \\
+\beta_{n}^{\prime} a_{1}^{2 n-1} y_{n}=\sum_{j}^{n} \beta_{k}^{\prime} a_{1}^{2(k-1)} \sum_{j=1}^{n+1-k} a_{i} y_{k+j-1} .
\end{gathered}
$$

From which, comparing the coefficients we have the following restrictions:

$$
b_{n+1} \beta_{j}=a_{1}^{2 j-3} \beta_{j}^{\prime}, \quad\left[\frac{n+4}{2}\right] \leq j \leq n .
$$


Consider the following product on the one hand:

$$
\begin{gathered}
{\left[y_{1}^{\prime}, y_{n+1}^{\prime}\right]=\left[a_{1} y_{1}+a_{2} y_{2}+\ldots+a_{n+1} y_{n+1}, b_{n} y_{n}+b_{n+1} y_{n+1}\right]=} \\
=-2 a_{1} b_{n+1} \sum_{k=\left[\frac{n+4}{2}\right]}^{n} \beta_{k} x_{k-1}+a_{1} b_{n+1} \beta x_{n}-2 b_{n+1} \sum_{i=2}^{\left[\frac{n+1}{2}\right]} a_{i} \sum_{k=\left[\frac{n+4}{2}\right]}^{n+2-i} \beta_{k} x_{k+i-2}+a_{n+1} b_{n+1} \gamma x_{n}
\end{gathered}
$$

and on the other hand, let us consider the followings product in the case of an odd $n$.

$$
\begin{gathered}
{\left[y_{1}^{\prime}, y_{n+1}^{\prime}\right]=-2 \sum_{k=\left[\frac{n+4}{2}\right]}^{n} \beta_{k}^{\prime} x_{k-1}^{\prime}+\beta^{\prime} x_{n}^{\prime}=-2 \beta_{\left[\frac{n+4}{2}\right]}^{\prime}\left(a_{1}^{2\left(\left[\frac{n+4}{2}\right]-1\right)} x_{\left[\frac{n+4}{2}\right]-1}+\right.} \\
\left.+a_{1}^{2\left(\left[\frac{n+4}{2}\right]-2\right)+1} a_{2} x_{\left[\frac{n+4}{2}\right]}+\ldots+a_{1}^{2\left(\left[\frac{n+4}{2}\right]-2\right)+1} a_{n-\left[\frac{n+4}{2}\right]+2} x_{n}-2 a_{1}^{2\left[\frac{n-1}{2}\right]+1} a_{n+1} \beta_{\left[\frac{n+4}{2}\right]} x_{n}\right)- \\
-2 \beta_{\left[\frac{n+4}{2}\right]+1}^{\prime}\left(a_{1}^{2\left[\frac{n+4}{2}\right]} x_{\left[\frac{n+4}{2}\right]}+a_{1}^{2\left(\left[\frac{n+4}{2}\right]-1\right)+1} a_{2} x_{\left[\frac{n+4}{2}\right]+1}+\ldots+a_{1}^{2\left(\left[\frac{n+4}{2}\right]-1\right)+1} a_{n-\left[\frac{n+4}{2}\right]+1} x_{n}\right)- \\
-\ldots-2 \beta_{n}^{\prime}\left(a_{1}^{2 n-2} x_{n-1}+a_{1}^{2 n-3} a_{2} x_{n}\right)+\beta^{\prime} a_{1}^{2 n} x_{n}=-2 \sum_{k=\left[\frac{n+4}{2}\right]}^{n} \beta_{k}^{\prime} a_{1}^{2 k-3} \sum_{i=1}^{n-k+2} a_{i} x_{k+i-2}+ \\
+4 \beta_{\left[\frac{n+4}{2}\right]}^{\prime} a_{1}^{2\left[\frac{n-1}{2}\right]+1} a_{n+1} \beta_{\left[\frac{n+4}{2}\right]} x_{n}+\beta^{\prime} a_{1}^{2 n} x_{n} .
\end{gathered}
$$

In the case of an even $n$, for the product $\left[y_{1}^{\prime}, y_{n+1}^{\prime}\right]$ we have:

$$
\begin{gathered}
{\left[y_{1}^{\prime}, y_{n+1}^{\prime}\right]=-2 \sum_{k=\left[\frac{n+4}{2}\right]}^{n} \beta_{k}^{\prime} x_{k-1}^{\prime}+\beta^{\prime} x_{n}^{\prime}=-2 \beta_{\left[\frac{n+4}{2}\right]}^{\prime}\left(a_{1}^{2\left(\left[\frac{n+4}{2}\right]-1\right)} x_{\left[\frac{n+4}{2}\right]-1}+\right.} \\
\left.+a_{1}^{2\left(\left[\frac{n+4}{2}\right]-2\right)+1} a_{2} x_{\left[\frac{n+4}{2}\right]}+\ldots+a_{1}^{2\left(\left[\frac{n+4}{2}\right]-2\right)+1} a_{n-\left[\frac{n+4}{2}\right]+1} x_{n}\right)-2 \beta_{\left[\frac{n+4}{2}\right]+1}^{\prime}\left(a_{1}^{2\left[\frac{n+4}{2}\right]} x_{\left[\frac{n+4}{2}\right]}+\right. \\
\left.+a_{1}^{2\left(\left[\frac{n+4}{2}\right]-1\right)+1} a_{2} x_{\left[\frac{n+4}{2}\right]+1}+\ldots+a_{1}^{2\left(\left[\frac{n+4}{2}\right]-1\right)+1} a_{n-\left[\frac{n+4}{2}\right]+1} x_{n}\right)-\ldots-2 \beta_{n}^{\prime}\left(a_{1}^{2 n-2} x_{n-1}+\right. \\
\left.+a_{1}^{2 n-3} x_{n}\right)+\beta^{\prime} a_{1}^{2 n} x_{n}=-2 \sum_{k=\left[\frac{n+4}{2}\right]}^{n} \beta_{k}^{\prime} a_{1}^{2 k-3} \sum_{i=1}^{n-k+2} a_{i} x_{k+i-2}+\beta^{\prime} a_{1}^{2 n} x_{n} .
\end{gathered}
$$

Comparing the coefficients we obtain the following restrictions:

when $n$ is odd

$$
\left\{\begin{array}{l}
b_{n+1} \beta_{j}=a_{1}^{2 j-3} \beta_{j}^{\prime}, \quad\left[\frac{n+4}{2}\right] \leq j \leq n, \\
a_{n+1} b_{n+1} \gamma+a_{1} b_{n+1} \beta=a_{1}^{2 n} \beta^{\prime}+4 \beta_{\left[\frac{n+4}{2}\right]}^{\prime} a^{2\left[\frac{n+1}{2}\right]-1} a_{n+1} \beta_{\left[\frac{n+4}{2}\right]}
\end{array}\right.
$$


when $n$ is even

$$
\left\{\begin{array}{l}
b_{n+1} \beta_{j}=a_{1}^{2 j-3} \beta_{j}^{\prime}, \quad\left[\frac{n+4}{2}\right] \leq j \leq n, \\
a_{n+1} b_{n+1} \gamma+a_{1} b_{n+1} \beta=a_{1}^{2 n} \beta^{\prime}
\end{array}\right.
$$

It is not difficult to check that considering other multiplications we have either restrictions (3.7)-(3.8) or identity.

Note that from (3.6) we have $b_{n}=\frac{-a_{n+1} b_{n+1} \gamma}{a_{1}}$.

Thus, combining the restrictions (3.6), (3.7) and (3.8) it follows the proof of the theorem.

Introduce the operators which are similar like $k$-dimensional vectors:

$$
\begin{aligned}
& j \\
& V_{j, k}^{0}\left(\alpha_{1}, \alpha_{2}, \ldots, \alpha_{k}\right)=\left(0, \ldots, 0,1, \quad \delta \sqrt[j]{\delta^{j+1}} S_{m, j}^{j+1} \alpha_{j+1}, \delta \sqrt[j]{\delta^{j+2}} S_{m, j}^{j+2} \alpha_{j+2}, \ldots, \delta \sqrt[j]{\delta^{k}} S_{m, j}^{k} \alpha_{k}\right) ; \\
& j \\
& V_{j, k}^{1}\left(\alpha_{1}, \alpha_{2}, \ldots, \alpha_{k}\right)=\left(0, \ldots, 0,1, \quad S_{m, j}^{j+1} \alpha_{j+1}, S_{m, j}^{j+2} \alpha_{j+2}, \ldots, S_{m, j}^{k} \alpha_{k}\right) ; \\
& j \\
& V_{j, k}^{2}\left(\alpha_{1}, \alpha_{2}, \ldots, \alpha_{k}\right)=\left(0, \ldots, 0,1, \quad S_{m, 2 j+1}^{2(j+1)+1} \alpha_{j+1}, S_{m, 2 j+1}^{2(j+2)+1} \alpha_{j+2}, \ldots, S_{m, 2 j+1}^{2 k+1} \alpha_{k}\right) ; \\
& V_{k+1, k}^{0}\left(\alpha_{1}, \alpha_{2}, \ldots, \alpha_{k}\right)=V_{k+1, k}^{1}\left(\alpha_{1}, \alpha_{2}, \ldots, \alpha_{k}\right)=V_{k+1, k}^{2}\left(\alpha_{1}, \alpha_{2}, \ldots, \alpha_{k}\right)=(0,0, \ldots, 0) \text {; } \\
& j \\
& W_{s, k}\left(0, \ldots, 0,1, \quad S_{m, j}^{j+1} \alpha_{j+1}, S_{m, j}^{j+2} \alpha_{j+2}, \ldots, S_{m, j}^{k} \alpha_{k}\right)= \\
& j \quad s+j \\
& =\left(0, \ldots, 0,1, \quad 0, \ldots, 0, \quad 1, \quad S_{m, j}^{j+1} \alpha_{s+j+1}, S_{m, j}^{j+2} \alpha_{s+j+2}, \ldots, S_{m, j}^{k-s} \alpha_{k}\right), \\
& W_{k+1-j, k}\left(0, \ldots, 0,{ }_{1}^{j}, 0, \ldots, 0\right)=(0, \ldots, 0,1,0, \ldots, 0)
\end{aligned}
$$

where $k \in \mathbb{C}, \delta= \pm 1,1 \leq j \leq k, 1 \leq s \leq k-j, S_{m, t}=\cos \frac{2 \pi m}{t}+i \sin \frac{2 \pi m}{t}(m=$ $0,1, \ldots, t-1)$.

Theorem 3.2 allows us to classify the Leibniz superalgebras from the variety Leib ${ }^{n, m}$ with characteristic sequence $(n \mid m-1,1)$, nilindex $n+m$ and $m=n+1$.

Theorem 3.3. Let $L$ be a Leibniz superalgebra of variety Leib ${ }^{n, m}$ with characteristic sequence $(n \mid m-1,1)$, nilindex $n+m$ and $m=n+1$. Then $L$ is isomorphic to one of the following pairwise non isomorphic superalgebras:

if $n$ is odd (i.e. $n=2 q-1$ ):

$$
\begin{array}{lll}
L\left(1, \delta \beta_{q+1}, V_{j, q-2}^{0}\left(\beta_{q+2}, \beta_{q+3}, \ldots, \beta_{n}\right), 0\right), & \beta_{q+1} \neq \pm \frac{1}{2}, & 1 \leq j \leq q-1, \\
L\left(1, \beta_{q+1}, V_{j, q-1}^{0}\left(\beta_{q+2}, \beta_{q+3}, \ldots, \beta_{n}, \beta\right)\right), & \beta_{q+1}= \pm \frac{1}{2}, \quad 1 \leq j \leq q, \\
L\left(0,1, V_{j, q-2}^{0}\left(\beta_{q+2}, \beta_{q+3}, \ldots, \beta_{n}\right), 0\right), & 1 \leq j \leq q-1, & \\
L\left(0,0, W_{s, q-1}\left(V_{j, q-1}^{1}\left(\beta_{q+2}, \beta_{q+3}, \ldots, \beta_{n}, \beta\right)\right)\right), & 1 \leq j \leq q-1, \quad 1 \leq s \leq q-j, \\
L(0,0, \ldots, 0) ; &
\end{array}
$$


if $n$ is even (i.e. $n=2 q$ ):

$$
\begin{array}{ll}
L\left(1, V_{j, q-1}^{2}\left(\beta_{q+2}, \beta_{q+3}, \ldots, \beta_{n},\right), 0\right), & 1 \leq j \leq q \\
L\left(0, W_{s, q}\left(V_{j, q}^{1}\left(\beta_{q+2}, \beta_{q+3}, \ldots, \beta_{n}, \beta\right)\right)\right), & 1 \leq j \leq q, \quad 1 \leq s \leq q+1-j, \\
L(0,0, \ldots, 0) . &
\end{array}
$$

Proof. Consider $n$ is odd, i.e. $n=2 q-1$, where $q \in \mathbb{N}$. From Theorem 3.2 we have the following restrictions:

$$
\left\{\begin{array}{l}
b_{n+1}^{2} \gamma=\gamma^{\prime} a_{1}^{2 n} \\
b_{n+1} \beta_{j}=a_{1}^{2 j-3} \beta_{j}^{\prime}, \quad q+1 \leq j \leq n \\
a_{n+1} b_{n+1} \gamma+a_{1} b_{n+1} \beta=a_{1}^{2 n} \beta^{\prime}+4 \beta_{q+1}^{\prime} a_{1}^{n} a_{n+1} \beta_{q+1}
\end{array}\right.
$$

for which we consider all possible cases.

Case 1. Let $\gamma \neq 0$. Then taking $b_{n+1}= \pm \frac{a_{1}^{n}}{\sqrt{\gamma}}$, we obtain $\gamma^{\prime}=1$. Substituting the value of $b_{n+1}$ in other restrictions we obtain equalities:

$$
\begin{gathered}
\beta_{q+1+j}^{\prime}= \pm \frac{\beta_{q+1+j}}{a_{1}^{2 j} \sqrt{\gamma}}, \quad 0 \leq j \leq q-2, \\
\beta^{\prime}= \pm \frac{a_{n+1}\left(\gamma-4 \beta_{q+1}^{2}\right)+a_{1} \beta}{a_{1}^{n} \sqrt{\gamma}} .
\end{gathered}
$$

Case 1.1. If $\gamma-4 \beta_{q+1}^{2} \neq 0$, then putting $a_{n+1}=-\frac{a_{1} \beta}{\gamma-4 \beta_{q+1}^{2}}$, we have $\beta^{\prime}=0$ and $\beta_{q+1+j}^{\prime}= \pm \frac{\beta_{q+1+j}}{\sqrt{\gamma}} a_{1}^{-2 j}$ for $0 \leq j \leq q-2$.

If $\beta_{q+1+j}=0$ for any $j \in\{1, \ldots, q-2\}$, then $\beta_{q+1+j}^{\prime}=0$ and we obtain the superalgebras:

$$
L\left(1, \delta \beta_{q+1}, 0, \ldots, 0\right), \quad \delta= \pm 1 .
$$

If $\beta_{q+2}=\beta_{q+3}=\ldots=\beta_{q+t}=0$ and $\beta_{q+t+1} \neq 0$ for some $t \in\{1,2, \ldots, q-2\}$. Then taking $a_{1}^{-2 t}= \pm \frac{\sqrt{\gamma}}{\beta_{q+t+1}}$ (i.e. $a_{1}^{-2}=\sqrt[t]{ \pm 1} \sqrt[t]{\left|\frac{\sqrt{\gamma}}{\beta_{q+t+1}}\right|}\left(\cos \frac{\varphi}{t}+i \sin \frac{\varphi}{t}\right)\left(\cos \frac{2 \pi m}{t}+i \sin \frac{2 \pi m}{t}\right)$, where $\left.\varphi=\arg \left(\frac{\sqrt{\gamma}}{\beta_{q+t+1}}\right), m=0,1, \ldots, t-1\right)$, we obtain:

$$
\beta_{q+t+1}^{\prime}=1 \text { and } \beta_{q+t+j}^{\prime}= \pm \sqrt[t]{ \pm 1} S_{m, t}^{j} \beta_{q+t+j}, \quad m=0,1, \ldots, t-1
$$

So, in this case we have the following superalgebras:

$$
L\left(1, \delta \beta_{q+1}, V_{j, q-2}^{0}\left(\beta_{q+2}, \beta_{q+3}, \ldots, \beta_{n}\right), 0\right), \quad \beta_{q+1} \neq \pm \frac{1}{2}, \quad 1 \leq j \leq q-1, \quad \delta= \pm 1 .
$$

Case 1.2. If $\gamma-4 \beta_{q+1}^{2}=0$, then $\beta_{q+1}^{\prime}= \pm \frac{1}{2}$ and we have

$$
\beta_{q+1+j}^{\prime}= \pm \frac{\beta_{q+1+j}}{\sqrt{\gamma}} a_{1}^{-2 j}, \quad 1 \leq j \leq q-2,
$$




$$
\beta^{\prime}= \pm \frac{\beta}{\sqrt{\gamma}} a_{1}^{-n+1}
$$

If we assume that $j=q-1$ in restriction (3.9), then we obtain $\beta_{2 q}^{\prime}= \pm \frac{\beta_{2 q}}{\sqrt{\gamma}} a_{1}^{-2(q-1)}$. Since $n=2 q-1$, then $-2(q-1)=-n+1$, i.e. we formally have restriction (3.10) and therefore restriction (3.10) can be considered as a particular case of restriction (3.9) when $j=q-1$.

Furthermore, as in case 1.1, we obtain the following superalgebras:

$$
L\left(1, \beta_{q+1}, V_{j, q-1}^{0}\left(\beta_{q+2}, \beta_{q+3}, \ldots, \beta_{n}, \beta\right)\right), \quad \beta_{q+1}= \pm \frac{1}{2}, \quad 1 \leq j \leq q .
$$

Case 2. $\gamma=0$. Then $\gamma^{\prime}=0$ and

$$
\begin{gathered}
b_{n+1} \beta_{q+1+j}=a_{1}^{2 q-1+2 j} \beta_{q+1+j}^{\prime}, \quad 0 \leq j \leq q-2, \\
a_{1} b_{n+1} \beta=a_{1}^{2 n} \beta^{\prime}+4 \beta_{q+1}^{\prime} a_{1}^{n} a_{n+1} \beta_{q+1} .
\end{gathered}
$$

Case 2.1. $\beta_{q+1} \neq 0$. Then taking $b_{n+1}=\frac{a_{1}^{n}}{\beta_{q+1}}$ and $a_{n+1}=\frac{b_{n+1} \beta}{4 a_{1}^{n-1} \beta_{q+1}}$, we have $\beta_{q+1}^{\prime}=0, \beta^{\prime}=0$ and $\beta_{q+1+j}^{\prime}=\frac{\beta_{q+1+j}}{\beta_{q+1}} a_{1}^{-2 j}, 1 \leq j \leq q-2$.

Furthermore, as in case 1.1, we obtain the superalgebras:

$$
L\left(0,1, V_{j, q-2}^{1}\left(\beta_{q+2}, \beta_{q+3}, \ldots, \beta_{n}\right), 0\right), \quad 1 \leq j \leq q-1 .
$$

Case 2.2. $\beta_{q+1}=0$. Then $\beta_{q+1}^{\prime}=0$ and

$$
\begin{gathered}
b_{n+1} \beta_{q+1+j}=a_{1}^{2 q-1+2 j} \beta_{q+1+j}^{\prime}, \quad 1 \leq j \leq q-2, \\
b_{n+1} \beta=a_{1}^{2 n-1} \beta^{\prime} .
\end{gathered}
$$

If we assume that $j=q-1$, in restriction (3.11), then we obtain $b_{n+1} \beta_{2 q}=a_{1}^{2 n-1} \beta_{2 q}^{\prime}$. Since $n=2 q-1$, then $2 q-1+2(q-1)=4 q-3=2 n-1$, and then we formally obtain restriction (3.12) and, therefore restriction (3.12) can be considered as a particular case of (3.11) when $j=q-1$.

Case 2.2.1 $\beta_{q+2}=\beta_{q+3}=\ldots=\beta_{q+t}=0$ and $\beta_{q+t+1} \neq 0$ for some $t \in\{1,2, \ldots, q-$ $1\}$. Then if we choose $b_{n+1}=\frac{a_{1}^{2 q-1+2 t}}{\beta_{q+1+t}}$, we obtain $\beta_{q+1+t}^{\prime}=1$ and

$$
\beta_{q+1+j}^{\prime}=\frac{\beta_{q+1+j}}{\beta_{q+1+t}} a_{1}^{-2(j-t)}, \quad t+1 \leq j \leq q-1 .
$$

Thus, in this case we have the superalgebras:

$$
L\left(0,0, W_{s, q-1}\left(V_{j, q-1}^{1}\left(\beta_{q+2}, \beta_{q+3}, \ldots, \beta_{n}, \beta\right)\right)\right), \quad 1 \leq j \leq q-1, \quad 1 \leq s \leq q-j .
$$

Case 2.2.2 $\beta_{q+j+1}=0$ for any $j(1 \leq j \leq q-1)$. Then we obtain superalgebra:

$$
L(0,0, \ldots, 0) \text {. }
$$


Consider the case of even $n$, i.e. $n=2 q$ for some $q \in \mathbb{N}$.

Then from Theorem 3.2 we have the following restrictions:

$$
\left\{\begin{array}{l}
b_{n+1}^{2} \gamma=\gamma^{\prime} a_{1}^{2 n}, \\
b_{n+1} \beta_{q+2+j}=a_{1}^{2 q+2 j+1} \beta_{q+2+j}^{\prime}, \quad 0 \leq j \leq q-2, \\
a_{n+1} b_{n+1} \gamma+a_{1} b_{n+1} \beta=a_{1}^{2 n} \beta^{\prime} .
\end{array}\right.
$$

Case 1. $\gamma \neq 0$. Then taking $b_{n+1}= \pm \frac{a_{1}^{n}}{\sqrt{\gamma}}$ and $a_{n+1}=-\frac{a_{1} \beta}{\gamma}$ we obtain $\gamma^{\prime}=1$; $\beta_{q+2+j}^{\prime}= \pm \frac{\beta_{q+2+j}}{a_{1}^{2 j+1} \sqrt{\gamma}}(0 \leq j \leq q-2)$ and $\beta^{\prime}=0$.

If $\beta_{q+2+j}=0$ for any $j(0 \leq j \leq q-2)$, then we have superalgebra:

$$
L(1,0, \ldots, 0) \text {. }
$$

If $\beta_{q+2}=\beta_{q+3}=\ldots=\beta_{q+t+1}=0$ and $\beta_{q+t+2} \neq 0$ for some $t(1 \leq t \leq q-2)$, then putting $a_{1}^{-(2 t+1)}= \pm \frac{\sqrt{\gamma}}{\beta_{q+2+t}}$ (i.e. $a_{1}^{-1}=$ $\pm \sqrt[2 t+1]{\left|\frac{\sqrt{\gamma}}{\beta_{q+2+t}}\right|}\left(\cos \frac{\varphi}{2 t+1}+i \sin \frac{\varphi}{2 t+1}\right)\left(\cos \frac{2 \pi m}{2 t+1}+i \sin \frac{2 \pi m}{2 t+1}\right)$, where $\varphi=\arg \left(\frac{\sqrt{\gamma}}{\beta_{q+2+t}}\right), m=$ $0,1, \ldots, 2 t)$, and substituting the value of $a_{1}^{-1}$ in other restrictions we obtain

$$
\begin{aligned}
& \beta_{q+2+j}^{\prime}= \pm \frac{\beta_{q+2+j}}{\sqrt{\gamma}}\left( \pm \sqrt[2 t+1]{\left|\frac{\sqrt{\gamma}}{\beta_{q+2+j}}\right|}\left(\cos \frac{\varphi}{2 t+1}+i \sin \frac{\varphi}{2 t+1}\right) S_{m, 2 j+1}\right)^{2 j+1}= \\
& =\beta_{q+2+j} S_{m, 2 j+1}^{2 j+1}, \quad t+1 \leq j \leq q-2 .
\end{aligned}
$$

Thus, in this case we have the following superalgebras:

$$
L\left(1, V_{j, q-1}^{2}\left(\beta_{q+2}, \beta_{q+3}, \ldots, \beta_{n},\right), 0\right), \quad 1 \leq j \leq q .
$$

Case 2. $\gamma=0$. Then $\gamma^{\prime}=0$ and $\beta_{j+2+q}^{\prime}=\frac{b_{n+1} \beta_{q+2+j}}{a_{1}^{2 q+1+2 j}}, 0 \leq j \leq q-2, \beta^{\prime}=\frac{b_{n+1} \beta}{a_{1}^{2 n-1}}$.

Note that in this case $\beta^{\prime}$ also can be considered as a particular case of $\beta_{j+2+q}^{\prime}$ for $j=q-1$.

If $\beta_{q+2+j}=0$ for any $j(0 \leq j \leq q-1)$, then we have the superalgebra:

$$
L(0,0,0, \ldots, 0) \text {. }
$$

If $\beta_{q+2}=\beta_{q+3}=\ldots=\beta_{q+t+1}=0$ and $\beta_{q+t+2} \neq 0$ for some $t(1 \leq t \leq q-1)$. Then putting $b_{n+1}=\frac{a_{1}^{2 q+2 t+1}}{\beta_{q+2+t}}$, we obtain:

$$
\beta_{q+2+t}^{\prime}=1, \quad \beta_{q+2+j}^{\prime}=\frac{\beta_{q+2+j}}{\beta_{q+2+t}} a_{1}^{-2(j-t)} \quad(t+1 \leq j \leq q-1) .
$$

As in case 2.2.1 for odd $n$, we obtain superalgebras:

$$
L\left(0, W_{s, q}\left(V_{j, q}^{1}\left(\beta_{q+2}, \beta_{q+3}, \ldots, \beta_{n}, \beta\right)\right)\right), \quad 1 \leq j \leq q, \quad 1 \leq s \leq q+1-j .
$$


The existence of an adapted basis under the conditions of Lemma 3.1 for $m=n+2$ is represented in the following lemma.

Lemma 3.3. Let $L$ be a Leibniz superalgebra of variety Leib ${ }^{n, m}$ with characteristic sequence $(n \mid m-1,1)$, nilindex $n+m$ and $m=n+2$. Then, there exists a basis $\left\{x_{1}, x_{2}, \ldots, x_{n}, y_{1}, y_{2}, \ldots, y_{n+2}\right\}$ of $L$ in which the multiplication has the following form:

$$
\begin{gathered}
{\left[x_{i}, x_{1}\right]=x_{i+1}, 1 \leq i \leq n-1, \quad\left[y_{j}, x_{1}\right]=y_{j+1}, 1 \leq j \leq n,} \\
{\left[x_{i}, y_{1}\right]=\frac{1}{2} y_{i+1}, 1 \leq i \leq n, \quad\left[y_{j}, y_{1}\right]=x_{j}, 1 \leq j \leq n,} \\
{\left[x_{i}, y_{n+2}\right]=\sum_{k=\left[\frac{n+5}{2}\right]}^{n+2-i} \beta_{k} y_{k-1+i}, 1 \leq i \leq\left[\frac{n}{2}\right],\left[y_{j}, y_{n+2}\right]=-2 \sum_{k=\left[\frac{n+5}{2}\right]}^{n+2-j} \beta_{k} x_{k-2+j}, 1 \leq j \leq\left[\frac{n}{2}\right] .}
\end{gathered}
$$

Proof. The proof of this lemma is analogous to the proof of Lemma 3.2. $\left.\beta_{n+1}\right)$.

Let us denote the superalgebra from the family of Lemma $3.3 \mathrm{by} L\left(\beta_{\left[\frac{n+5}{2}\right]}, \beta_{\left[\frac{n+5}{2}\right]+1}, \ldots\right.$,

The condition of isomorphism of two superalgebras is represented in the following theorem.

Theorem 3.4. Two superalgebras $L\left(\beta_{\left[\frac{n+5}{2}\right]}, \beta_{\left[\frac{n+5}{2}\right]+1}, \ldots, \beta_{n+1}\right)$ and $L^{\prime}\left(\beta_{\left[\frac{n+5}{2}\right]}^{\prime}, \beta_{\left[\frac{n+5}{2}\right]+1}^{\prime}, \ldots, \beta_{n+1}^{\prime}\right)$ are isomorphic if and only if there exist $a_{1}, b_{n+2} \in \mathbb{C}$ such that the following conditions hold:

$$
b_{n+2} \beta_{j}=a_{1}^{2 j-3} \beta_{j}^{\prime}, \quad\left[\frac{n+5}{2}\right] \leq j \leq n+1 .
$$

Proof. By a change of basis the generators of the new basis are expressed by

$$
y_{1}^{\prime}=\sum_{i=1}^{n+2} a_{i} y_{i}, \quad y_{n+1}^{\prime}=\sum_{j=1}^{n+2} b_{j} y_{j}
$$

where the $\operatorname{rank}\left(\begin{array}{cccc}a_{1} & a_{2} & \ldots & a_{n+2} \\ b_{1} & b_{2} & \ldots & b_{n+2}\end{array}\right)=2$, this alows us to express the elements of the new basis $\left\{x_{1}^{\prime}, x_{2}^{\prime}, \ldots, x_{n}^{\prime}, y_{1}^{\prime}, y_{2}^{\prime}, \ldots, y_{n+2}^{\prime}\right\}$ of the superalgebra $L^{\prime}\left(\beta_{\left[\frac{n+5}{2}\right]}^{\prime}, \beta_{\left[\frac{n+5}{2}\right]+1}^{\prime}, \ldots, \beta_{n+1}^{\prime}\right)$ with respect to the elements of old basis $\left\{x_{1}, x_{2}, \ldots, x_{n}, y_{1}\right.$, $\left.y_{2}, \ldots, y_{n+2}\right\}$ as:

$$
\begin{gathered}
x_{1}^{\prime}=\left[y_{1}^{\prime}, y_{1}^{\prime}\right]=a_{1} \sum_{k=1}^{n} a_{k} x_{k}-2 a_{n+2} \sum_{i=1}^{\left[\frac{n}{2}\right]} a_{i} \sum_{k=\left[\frac{n+5}{2}\right]}^{n+2-i} \beta_{k} x_{k-2+i} ; \\
x_{t+1}^{\prime}=\left[x_{t}^{\prime}, x_{1}^{\prime}\right]=a_{1}^{2 t+1} \sum_{k=1}^{n-t} a_{k} x_{t+k}-2 a_{1}^{2 t} a_{n+2} \sum_{i=1}^{\left[\frac{n}{2}\right]-t} a_{i} \sum_{k=\left[\frac{n+5}{2}\right]}^{n+2-t-i} \beta_{k} x_{k+t-2+i}, \quad 1 \leq t \leq\left[\frac{n-2}{2}\right] ;
\end{gathered}
$$




$$
\begin{aligned}
& x_{t+1}^{\prime}=\left[x_{t}^{\prime}, x_{1}^{\prime}\right]=a_{1}^{2 t+1} \sum_{k=1}^{n-t} a_{k} x_{t+k}, \quad\left[\frac{n}{2}\right] \leq t \leq n-1 ; \\
& y_{t}^{\prime}=\left[y_{t-1}^{\prime}, x_{1}^{\prime}\right]=a_{1}^{2(t-1)} \sum_{i=1}^{n+2-t} a_{i} y_{t-1+i}, 2 \leq t \leq n+1 .
\end{aligned}
$$

If we consider the products

$$
\left[x_{i}^{\prime}, x_{1}^{\prime}\right]=\frac{1}{2} y_{i+1}^{\prime}, \quad 1 \leq i \leq n,\left[y_{t}^{\prime}, y_{1}^{\prime}\right]=x_{t}^{\prime}, \quad 1 \leq t \leq n
$$

we find no restrictions.

Consider the chain of the equalities:

$$
\left[y_{n+2}^{\prime}, y_{n+2}^{\prime}\right]=b_{1} \sum_{k=1}^{n} b_{k} x_{k}-2 b_{n+2} \sum_{i=1}^{\left[\frac{n}{2}\right]} b_{i} \sum_{k=\left[\frac{n+5}{2}\right]}^{n+2-i} \beta_{k} x_{k-2+i}=0 \text {. }
$$

Comparing the coefficients of the basis elements in the last equality we obtain the following restrictions:

$$
b_{i}=0,1 \leq i \leq\left[\frac{n}{2}\right]
$$

From the following equalities we obtain:

$$
\left[y_{n+2}^{\prime}, y_{1}^{\prime}\right]=\left[\sum_{i=\left[\frac{n}{2}\right]+1}^{n+2} b_{i} y_{i}, \sum_{j=1}^{n+2} a_{j} y_{j}\right]=a_{1} \sum_{k=\left[\frac{n}{2}\right]+1}^{n} b_{k} x_{k}=0
$$

restrictions and summing them with (3.13) we obtain $b_{i}=0,1 \leq i \leq n$.

Therefore we have $y_{n+2}^{\prime}=b_{n+1} y_{n+1}+b_{n+2} y_{n+2}$.

Consider the multiplications defining the parameters:

$$
\begin{gathered}
{\left[x_{1}^{\prime}, y_{n+2}^{\prime}\right]=\left[a_{1} \sum_{k=1}^{n} a_{k} x_{k}-2 a_{n+2} \sum_{i=1}^{\left[\frac{n}{2}\right]} a_{i} \sum_{k=\left[\frac{n+5}{2}\right]}^{n+2-i} \beta_{k} x_{k-2+i}, b_{n+1} y_{n+1}+b_{n+2} y_{n+2}\right]=} \\
=a_{1} b_{n+2} \sum_{j=1}^{\left[\frac{n-2}{2}\right]+1} a_{j} \sum_{k=\left[\frac{n+5}{2}\right]}^{n+2-j} \beta_{k} y_{k+j-1} ; \\
{\left[x_{1}^{\prime}, y_{n+2}^{\prime}\right]=\sum_{k=\left[\frac{n+5}{2}\right]}^{n+1} \beta_{k}^{\prime} y_{k}^{\prime}=\beta_{\left[\frac{n+5}{2}\right]}^{\prime} a_{1}^{2\left[\frac{n+5}{2}\right]-2}\left(a_{1} y_{\left[\frac{n+5}{2}\right]}+a_{2} y_{\left[\frac{n+5}{2}\right]+1}+\ldots+\right.} \\
\left.+a_{n+2-\left[\frac{n+5}{2}\right]} y_{n+1}\right)+b_{\left[\frac{n+5}{2}\right]+1}^{\prime} a_{1}^{2\left[\frac{n+5}{2}\right]}\left(a_{1} y\left[\frac{n+5}{2}\right]+1\right. \\
+\ldots+\beta_{n+1}^{\prime} a_{1}^{2 n+1} y_{n+1}=\sum_{k=\left[\frac{n+5}{2}\right]}^{n+1} \beta_{k}^{\prime} a_{1}^{2(k-1)} \sum_{j=1}^{n+2-k} a_{j} y_{k+j-1} .
\end{gathered}
$$


From (3.14) and (3.15) we obtain restrictions:

$$
b_{n+2} \beta_{j}=a_{1}^{2 j-1} \beta_{j}^{\prime}, \quad\left[\frac{n+5}{2}\right] \leq j \leq n+1 .
$$

If we consider other multiplications, then we obtain restrictions (3.16) or identities.

The description up to isomorphism of a family from Lemma 3.3 is represented in the following theorem.

Theorem 3.5. Let $L$ be a Leibniz superalgebra of variety Leib ${ }^{n, m}$ with characteristic sequence $(n \mid m-1,1)$, nilindex $n+m$ and $m=n+2$. Then $L$ is isomorphic to one of the following pairwise non isomorphic superalgebras:

$$
L\left(W_{s, n+2-\left[\frac{n+5}{2}\right]}\left(V_{j, n+2-\left[\frac{n+5}{2}\right]}^{1}\left(\beta_{\left[\frac{n+5}{2}\right]}, \beta_{\left[\frac{n+5}{2}\right]+1}, \ldots, \beta_{n+1}\right)\right)\right),
$$

where $1 \leq j \leq n+2-\left[\frac{n+5}{2}\right], 1 \leq s \leq n+3-\left[\frac{n+5}{2}\right]-j$

$$
L(0,0, \ldots, 0) \text {. }
$$

Proof. From Theorem 3.4 we have the following restrictions:

$$
b_{n+2} \beta_{\left[\frac{n+5}{2}\right]+j}^{\prime}=a_{1}^{2\left[\frac{n+5}{2}\right]+2 j-3} \beta_{\left[\frac{n+5}{2}\right]+j}^{\prime}, \quad 0 \leq j \leq n+1-\left[\frac{n+5}{2}\right] .
$$

As $\left[\frac{n+5}{2}\right] \approx q+2$, for $n=2 q$ or $n=2 q-1$, then we obtain:

$$
b_{n+2} \beta_{q+j+2}=a_{1}^{2 q+2 j+1} \beta_{q+j+2}^{\prime}, \quad 0 \leq j \leq n+1-q-2 .
$$

The proof of this theorem is complete by using the same arguments as in the proof of Theorem 3.3 for even case.

Thus, Theorems 3.3 and 3.5 complete the classifications (up to isomorphism) of Leibniz superalgebras with characteristic sequence $C(L)=(n \mid m-1,1)$ and nilindex $n+m$.

Acknowledgments. The last named author would like to acknowledge the hospitality of the University of Sevilla (Spain). He was supported by the grants INTAS - 04-83-3035 and NATO-Reintegration ref. CBP.EAP.RIG.983169.

\section{References}

[1] Albeverio S., Ayupov. Sh.A., Omirov B.A. On nilpotent and simple Leibniz algebras. Comm. in Algebra, 2005, vol. 33, No. 1, p. 159-172

[2] Ayupov Sh.A., Omirov B.A., On some classes of nilpotent Leibniz algebras. Sib. Math. J., 2001, vol. 42, No. 1, p. 18-29. 
[3] Ayupov Sh.A., Omirov B.A., Khudoyberdiyev A. Kh., The classification of filiform Leibniz superalgebras of nilindex $n+m$. Acta Mathematica Sinica, to appear 2009.

[4] Bordeman M., Gómez J.R., Khakimdjanov Yu., Navarro R.M., Some deformations of nilpotent Lie superalgebras. J. Geometry and Physics, (2007), 57(5), 1391-1403.

[5] Gilg M. On the classification of filiform Lie superalgebras. Proceeding of the I Colloquium on Lie Theory and Applications (Vigo), 2000, p. 83-87.

[6] Gilg M. Super-algébres: PhD thesis. University of Haute Alsace, 2000. 126 p.

[7] Gómez J.R., Navarro R.M., Omirov B.A. On nilpotent Leibniz superalgebras. Preprint MA1-02-XI06 Universidad de Sevilla, 2006, pp. 24 (arXiv.math.RA/0611723).

[8] Gómez J.R., Khakimdjanov Yu., Navarro R.M. Some problems concerning to nilpotent Lie superalgebras. J. Geom. Phys. (2004), vol. 51, No. 4, p. 473-486.

[9] Gómez J.R., Khakimdjanov Yu., Navarro R.M. Infinitesimal deformations of the Lie superalgebras. J. Geom. Phys. (2008), p. 849-859.

[10] Kac V.G. Lie superalgebras, Advances in Math. (1977), vol. 26(1), p. 8-96.

[11] Loday J.-L. Une version non commutative des algèbres de Lie: les algèbres de Leibniz. Enseign. Math., 1993, vol. 39, p. 269-293.

Luisa M. Camacho, José R. Gómez. Dpto. Matemática Aplicada I. Universidad de Sevilla. Avda. Reina Mercedes, s/n. 41012 Sevilla. (Spain), e-mail: lcamacho@us.es,jrgomez@us.es

Rosa M. Navarro. Dpto. de Matemáticas, Universidad de Extremadura, Cáceres (Spain), e-mail: rnavarro@unex.es

Baknrom A. Omirov. Institute of Mathematics and Information Technologues, Uzbekistan Academy of Science, F. Hodjaev str. 29, 100125, Tashkent (Uzbekistan), e-mail: omirovb@mail.ru 